\title{
Intrarenal Vascular Sites of Action of Adenosine and Glucagon
}

\author{
Yasuharu AKI, Tetsuo SHOJI, Kouichi HASUI, Kiyoshi FUKUI, \\ Toshiaki TAMAKI, Hiroshi IWAO and Youichi ABE \\ Department of Pharmacology. Kagawa Medical School. Kagawa 761-07. Japan
}

Accepted August 27. 1990

\begin{abstract}
Our purpose was to localize the intrarenal vascular sites of action of adenosine and glucagon. Renal blood flow (RBF) and glomerular filtration rate (GFR) were measured in anesthetized dogs, and renal perfusion pressure (RPP) was varied by an adjustable aortic clamp. At normal RPP, RBF was increased by all agents. In contrast, GFR was increased by glucagon, decreased by adenosine and unchanged by acetylcholine (ACh) or adenosine plus glucagon. The increases in RBF by glucagon occurred only at RPPs within the autoregulatory pressure range, and renal autoregulatory capability was attenuated during the infusion of glucagon. In contrast, adenosine increased RBF at RPPs both within and below the autoregulatory pressure range, and the autoregulatory capability was not perceptibly impaired. Superimposition of glucagon to adenosine caused further vasodilation, and the autoregulatory efficiency was completely attenuated. There was no difference between the RPP-RBF or RPP-GFR relations obtained during infusion of adenosine plus glucagon and $A C h$, which dilates both the afferent and efferent arterioles. It is generally accepted that afferent arteriolar resistance attains a minimum value at RPP near the lower limit of the autoregulatory range. Thus, our data indicate that glucagon and adenosine preferentially dilate the afferent arteriole and the efferent arteriole, respectively.
\end{abstract}

Adenosine has been noted as one of the endogenous modulators of renal hemodynamics $(1-3)$. In the kidney, there are two resistance vessels, the afferent arterioles and the efferent arterioles. Different reactions of each type of arteriole to endogenous and exogenous vasoactive agents regulate renal blood flow (RBF) and glomerular filtration rate (GFR). Tagawa and Vander (4) reported that intrarenal infusion of adenosine produced afferent arteriolar constriction and efferent arteriolar dilation. In contrast, Osswald et al. (5) showed that adenosine constricted the afferent arteriole in superficial nephrons using a micropuncture method, while Murray and Churchill (6) reported that adenosine dilated both arterioles in isolated rat kidneys. Thus, there is still no consensus as to the intrarenal vascular sites of action of adenosine. Glucagon has also been noted as an endogenous regulator of renal hemodynamics, especially at the postprandial state (7). We reported that intrarenal administration of glucagon increased RBF and GFR proportionally, indicating the preferential dilation of the afferent arteriole $(8,9)$. However, there is still no clear evidence concerning the intrarenal vascular sites of action of glucagon. It is important to clarify the intrarenal vascular sites of action of adenosine and glucagon for an evaluation of the roles of adenosine and glucagon as regulators of renal hemodynamics.

In the present study. we evaluated the vascular sites of action of adenosine and glucagon in the canine kidney utilizing the following approach. Renal autoregulation is a well-established physiological phenomenon in which RBF and GFR remain relatively constant over a wide range of perfusion pressures through changes in the resistance of the afferent arteriole (10-15). Also, it is generally accepted that the resistance of the preglomerular vessels attains a minimum value at the renal perfusion pressure (RPP) near the 
lower limit of the autoregulatory pressure range $(14,15)$. Therefore, if a vasodilator is unable to increase RBF at the lower limit of the autoregulatory pressure range, its site of action is presumably the afferent arteriole. In contrast, if a vasodilator increases RBF at reduced RPP, its site of action is presumably the efferent arteriole. Based on the above argument, pressure-RBF and pressure-GFR relations were obtained during infusion of adenosine and glucagon, or acetylcholine (ACh) which dilates both the afferent and the efferent arterioles $(14,16,17)$. In addition. the effects of adenosine in combination with glucagon and the effects of ACh on pressureRBF and pressure-GFR relations were compared.

\section{Materials and Methods}

All experiments were performed on mongrel dogs of either sex weighing 12-17 kg. Animals were anesthetized with pentobarbital sodium (30 mg/kg, i.v.), followed by a maintenance dose if necessary. Following tracheal intubation, each animal was ventilated mechanically with a respirator. Polyethylene catheters were placed in the right brachial artery and vein for arterial blood sampling and monitoring of systemic arterial pressure, and for infusion of saline and inulin (Sigma, St. Louis, MO). The left kidney was exposed through a retroperitoneal flank incision. The kidney was denervated by division of all visible periaterial nerve fibers and sharp dissection of tissue connected to the renal hilum cephalad to the renal artery. RBF was measured by an electromagnetic flowmeter (MFV-1200, Nihon Kohden, Japan). RPP was measured via a catheter inserted into the right femoral artery and advanced into the abdominal aorta just below the left renal artery. A stainless adjustable clamp was placed on the aorta just above the bifurcation of the left renal artery and used to vary RPP. RBF and RPP were continuously monitored and recorded on a polygraph (model No. 361, NEC San-ei, Japan). A small, curved $23-$ gauge needle was inserted into the left renal artery proximal to the flow probe for the intrarenal infusion of saline or agents. It was maintained patent by a continuous infusion of isotonic saline at a rate of $0.19 \mathrm{ml} / \mathrm{min}$. After surgical preparation was completed, intravenous infusion of isotonic saline at a rate of $2 \mathrm{ml} / \mathrm{min}$ was started. For the measurement of GFR, a priming dose of inulin $(100 \mathrm{mg} / \mathrm{kg}$ ) was given into the right brachial vein, followed by a continuous infusion at $100 \mathrm{mg} / \mathrm{kg}$ per hour to maintain a constant blood level of inulin. Systemic arterial blood was collected from the right brachial artery, and renal venous blood collected via a cannula introduced through the left spermatic or ovarian vein. GFR was calculated as follows: $G F R=$ (arterial plasma concentration of inulin-renal venous plasma concentration of inulin) $\times$ renal plasma flow/arterial plasma concentration of inulin. Renal plasma flow $=\mathrm{RBF} \times(1-$ hema tocrit). Inulin was determined by the method of Walser et al. (18). To compare the GFR value obtained from the renal extraction of inulin with the GFR value obtained from the urinary excretion of inulin, a polyethylene catheter was inserted into the left ureter for urine collection.

Five series of experiments were performed.

1) After stabilization of RPP, RBF and urine flow, the pressure-flow and pressure-GFR relations were obtained. RPP was sequentially altered in 5 steps as indicated in Fig. 1 : normal pressure and 100,75,60, and $45 \mathrm{mmHg}$. Each pressure was maintained for several min to allow a complete autoregulatory response. In each step, systemic arterial and renal venous blood samples were collected from the right branchial artery and from the left renal vein. These samples were used to determine GFR using arteriovenous inulin differences in order to minimize delays that are inherent with the clearance approach. The RPP was then returned to the control level, and the animals were left for 30 min to allow for stabilization of each parameter. Urine was then collected during two consecutive 10-min control clearance periods. At the midpoint of each period. samples of systemic arterial and renal venous blood were collected. An intrarenal infusion of adenosine was started at a rate of $20 \mu \mathrm{g} / \mathrm{kg} /$ $\min (n=12)$. Urine and blood samples were obtained during two 10-min clearance periods after the initiation of the adenosine infusion. The pressure-flow and pressure-GFR relations were then examined, and blood samples were collected at each pressure level. The 
pressure-GFR relations were obtained in 8 of 12 dogs.

2) Intrarenal infusion of glucagon was performed at a rate of $0.5 \mu \mathrm{g} / \mathrm{kg} / \mathrm{min}$ instead of adenosine as described in series $1 \quad(n=10)$. The pressure-GFR relations were obtained in 5 of 10 dogs.

3) ACh $(4 \mu \mathrm{g} / \mathrm{kg} / \mathrm{min})$ was infused in 8 dogs instead of adenosine as described in series 1 . The pressure-GFR relations were obtained in 5 of 8 dogs.

4) The combined effects of adenosine and glucagon were examined in 8 dogs. Following control measurements, adenosine alone was infused into the renal artery of 8 dogs at 20 $\mu \mathrm{g} / \mathrm{kg} / \mathrm{min}$ for $25 \mathrm{~min}$, and then glucagon 10.5 $\mu \mathrm{g} / \mathrm{kg} / \mathrm{min}$ ) was superimposed. Two sets of blood and urine samples were obtained during infusion of adenosine and adenosine plus glucagon. During the combined infusion of adenosine and glucagon, the pressure-flow and -GFR relations were obtained as described in series 1 . The pressure-GFR relations were obtained in 6 of 8 dogs.

5) To test the reproducibility of pressureflow and $-G F R$ relations, these relations were examined in the same animal three times at 60 min intervals $(n=7)$. No significant differences were observed.

Data are expressed as means \pm S.E. Differences within groups were determined with Student's $t$-test for paired data, and differences between groups were determined with the unpaired $t$-test. Unless otherwise indicated, probability values of less than 0.05 were accepted as statistically significant differences.

\section{Results}

Table 1 shows the effects of glucagon, adenosine, adenosine plus glucagon and $A C h$ on RBF, GFR, urine flow and filtration fraction at normal RPP. No test agent affected the systemic arterial pressure except in the case of glucagon infusion. Intrarenal infusion of glucagon at $0.5 \mu \mathrm{g} / \mathrm{kg} / \mathrm{min}$ resulted in significant increases of RBF and GFR with no change in filtration fraction. Urine flow increased significantly along with the increases in RBF and GFR. Adenosine at $20 \mu \mathrm{g} / \mathrm{kg} / \mathrm{min}$ caused a biphasic response in RBF as reported previously $(4,5,19,20)$. Following initiation of infusion, RBF decreased and then increased to about $40 \%$ above the control level within $5 \mathrm{~min}$ after the start of infusion. This high level of RBF continued thereafter during the infusion. The later renal vasodilation was similar in magnitude to that produced by glucagon, but GFR was decreased significantly. Therefore, filtration fraction decreased significantly. Urine flow showed a tendency to decrease, but this was not statistically significant. Superimposition of glucagon $(0.5$ $\mu \mathrm{g} / \mathrm{kg} / \mathrm{min}$ ) to the adenosine infusion produced a further increase in RBF and restored the GFR decreased by infusion of adenosine alone to the control level. Filtration fraction was increased by the superimposition of glucagon, but it was still lower than the control level. Urine flow increased above the control level. ACh infusion at $4 \mu \mathrm{g} / \mathrm{kg} / \mathrm{min}$ significantly increased RBF by the same magnitude as during infusion of adenosine plus glucagon, but did not affect GFR. Urine flow increased significantly, and filtration fraction decreased.

Effects of adenosine on autoregulation of RBF and GFR: Figure 1 shows the relationship between RPP and RBF or GFR both in the absence and the presence of adenosine. When RPP was reduced in steps from normal pressure to $75 \mathrm{mmHg}$, complete autoregulation of RBF was observed in the absence of adenosine. GFR remained constant between normal pressure and $100 \mathrm{mmHg}$, but decreased slightly when pressure was decreased to $75 \mathrm{mmHg}$. The infusion of adenosine resulted in a significant increase of RBF and a significant decrease of GFR, but RBF and GFR remained relatively constant within the autoregulatory pressure range. Adenosine induced a vasodilatory response that increased RBF at RPPs below as well as within the autoregulatory pressure range (Fig. 1). The percent increases in RBF at normal RAP and $75 \mathrm{mmHg}$ were $39 \pm 4$ and $32 \pm 4 \%$, respectively. There was no significant difference between these values. Thus, RBF autoregulatory capability was not perceptibly impaired by adenosine, but the autoregulatory plateau was shifted upward. Conversely. the autoregulatory plateau of GFR was shifted downward, but GFR autoregulatory capability was not similarly impaired (Fig. 1). 


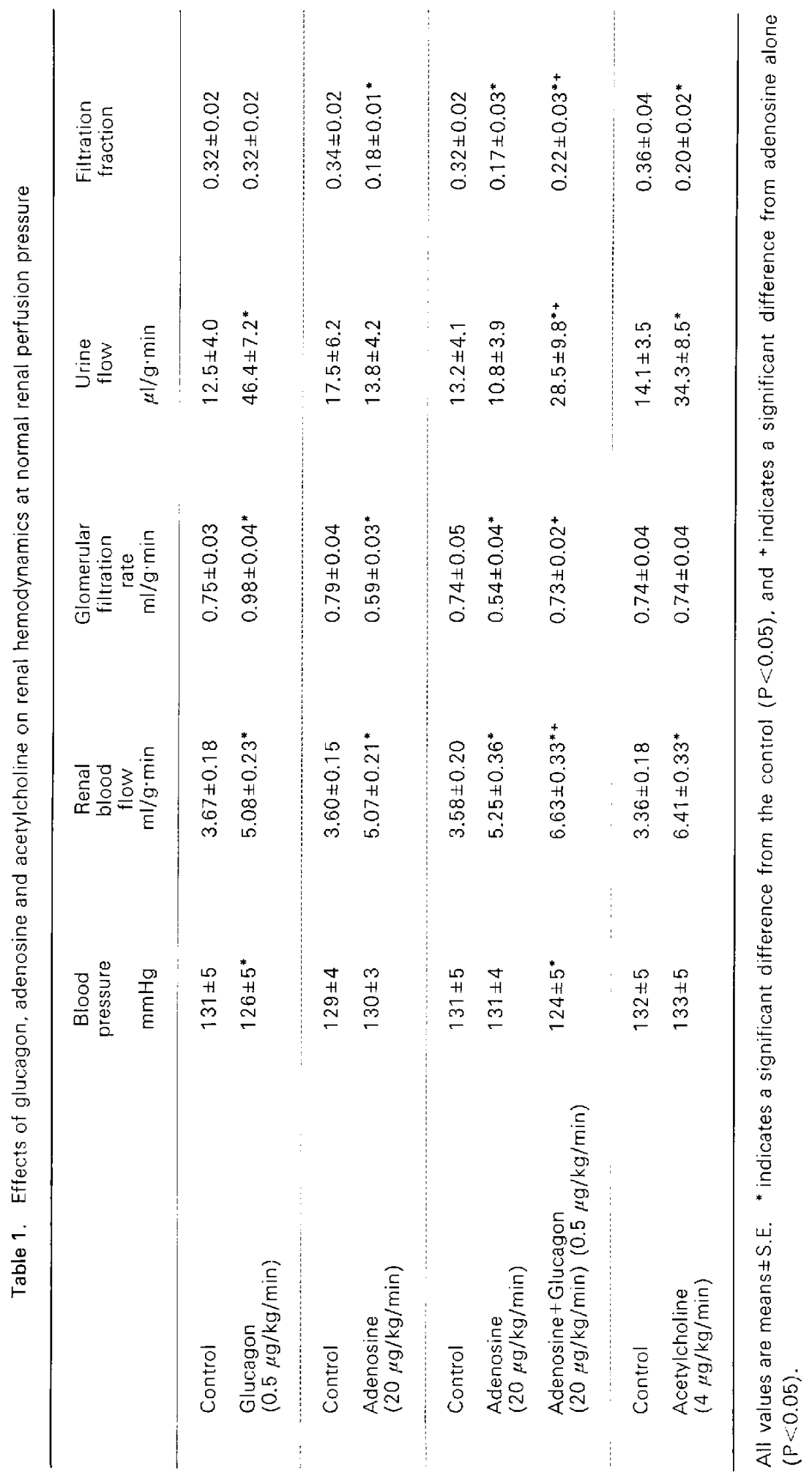




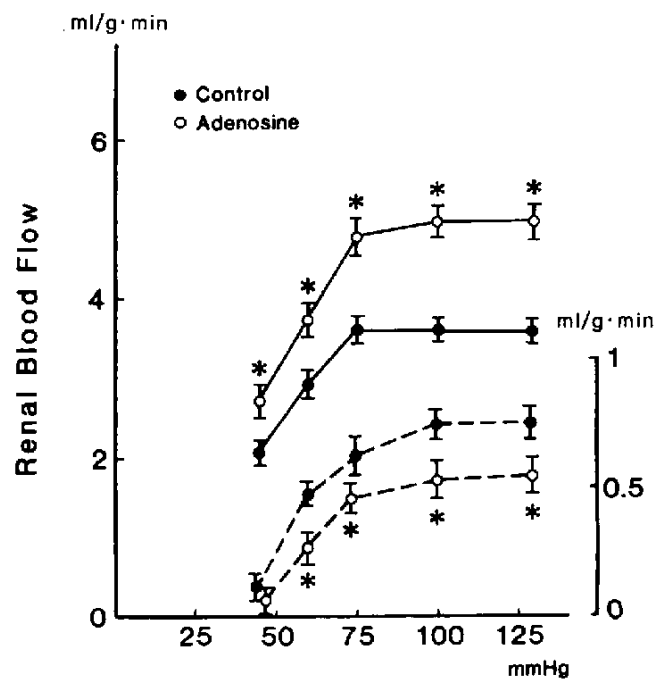

Renal Perfusion Pressure

Fig. 1. Relationships between renal perfusion pressure and renal blood flow (solid line, $n=12$ ) or glomerular filtration rate (dashed line, $n=8$ ) before (control) and during infusion of adenosine $(20 \mu \mathrm{g} /$ $\mathrm{kg} / \mathrm{min})$. * indicates a significant difference from the control at the same pressure $(P<0.05)$.

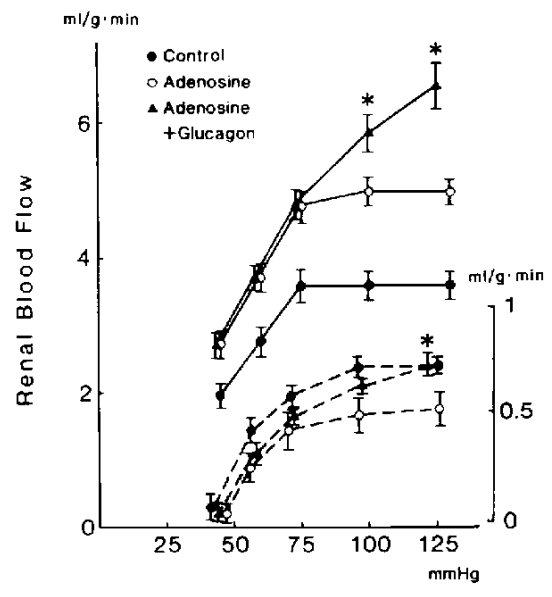

Renal Perfusion Pressure

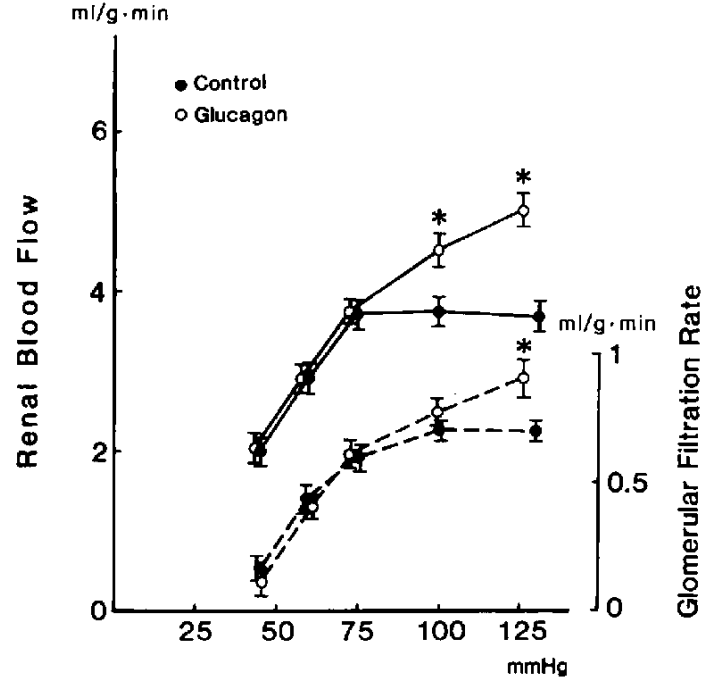

Renal Perfusion Pressure

Fig. 2. Relationships between renal perfusion pressure and renal blood flow (solid line. $n=10$ ) or glomerular filtration rate (dashed line, $n=5$ ) before (control) and during infusion of glucagon $(0.5 \mu \mathrm{g} /$ $\mathrm{kg} / \mathrm{min}) .{ }^{*}$ indicates a significant difference from the control at the same pressure $(P<0.05)$.

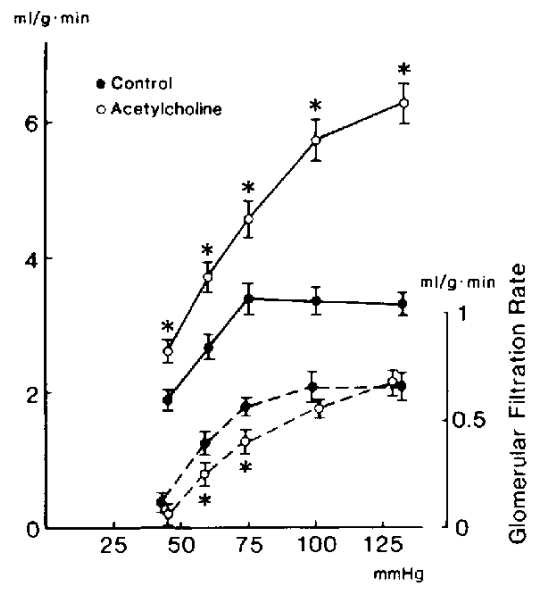

Renal Perfusion Pressure

Fig. 3. Relationships between renal perfusion pressure and renal blood flow (solid line, $n=8$ ) or glomerular filtration rate (dashed line, left panel, $n=6$; right panel, $n=5$ ) before (control) and during infusion of adenosine $(20 \mu \mathrm{g} / \mathrm{kg} / \mathrm{min})$ and superimposition of glucagon $(0.5 \mu \mathrm{g} / \mathrm{kg} / \mathrm{min})$ onto adenosine (left panel) and during infusion of acetylcholine ( $4 \mu \mathrm{g} / \mathrm{kg} / \mathrm{min}$ ) (right panel). *indicates a significant difference between adenosine alone and adenosine plus glucagon or between the control and $\mathrm{ACh}$ at the same pressure $(\mathrm{P}<0.05)$.

Effects of glucagon on autoregulation of RBF and GFR: Figure 2 shows the relationship between RPP and RBF or GFR in the absence and the presence of glucagon. When RPP was reduced in steps, autoregulation of RBF and GFR was also seen in the absence of 
glucagon. During infusion of glucagon, both RBF and GFR decreased in proportion to the reduction of RPP. The percent increases in RBF at normal RPP and $75 \mathrm{mmHg}$ during glucagon infusion were $38 \pm 3$ and $2 \pm 1$, respectively: and there was a significant difference between these changes $(P<0.01)$. At RPPs lower than the autoregulatory range, there was no significant difference between RBF or GFR in the absence and the presence of glucagon. That is, the vasodilation induced by glucagon was restricted to the RPPS within the autoregulatory pressure range (Fig. 2).

Effects of a combined infusion of adenosine and glucagon, and $\mathrm{ACh}$ on autoregulation of RBF and GFR: The left panel of Fig. 3 shows the RPP-RBF and -GFR relations under control conditions and during infusion of adenosine alone and adenosine plus glucagon. During adenosine infusion, both RBF and GFR were autoregulated as described above. During the superimposition of glucagon to adenosine infusion, both RBF and GFR decreased in proportion to the reduction of RPP. Thus, when glucagon was administered to dogs receiving adenosine, further vasodilation occurred at RPPs within the autoregulatory pressure range. However, there was no difference in RBF at RPPS lower than $75 \mathrm{mmHg}$ between infusion of adenosine alone and infusion of adenosine plus glucagon. The superimposition of glucagon onto adenosine dilated the renal blood vessels so that there was a marked attenuation of autoregulatory efficiency.

The right panel of Fig. 3 shows the relationships between RPP and RBF or GFR in the absence and the presence of $A C h$. During ACh infusion, both RBF and GFR decreased in proportion to the reduction of RPP. However, unlike glucagon, RBF during infusion of $\mathrm{ACh}$ was significantly higher than that in the absence of $\mathrm{ACh}$ at each pressure level. In contrast, GFR during infusion of $A C h$ was lower than that in the absence of ACh (Fig. 3). Thus, ACh induced a significant vasodilation that was characterized by a leftward shift in the RPP-RBF relationship. The percent increases in RBF during $A C h$ infusion at normal RPP and 75 $\mathrm{mmHg}$ were $93 \pm 8$ and $38 \pm 7 \%$, respectively.
Those during adenosine plus glucagon infusion at normal RPP and $75 \mathrm{mmHg}$ were $86 \pm 9$ and $37 \pm 7 \%$, respectively. Thus, the RBF responses at both RAPs were not different between $\mathrm{ACh}$ and adenosine plus glucagon. The RPP-GFR relationship during infusion of adenosine plus glucagon was also similar to that seen during infusion of $\mathrm{ACh}$ (Fig. 3). That is, RBF and GFR were decreased in proportion to the reduction of RPP, but RBF remained significantly higher than that before the infusion of drugs, even at RPPs below the lower limit of the autoregulatory pressure range.

\section{Discussion}

These experiments demonstrate that adenosine and glucagon increase RBF with a similar magnitude at normal perfusion pressure, but that there are clear-cut differences between RPP-RBF relations obtained during the infusion of these two agents. Glucagon markedly impaired autoregulatory efficiency and changed the nature of the pressure-flow relationship to one that nearly approached a passive linear system. Thus, the magnitude of the vasodilatory response induced by glucagon was highly dependent on the RPP within the autoregulatory pressure range. The responses obtained by the infusion of adenosine alone were consistent with the responses previously reported (21). In contrast to the effects of glucagon, we demonstrated that with adenosine, the autoregulatory capability was well-preserved, but at a higher plateau and with a upward shift in the passive portion of the relation. That is, the adenosine-induced renal vasodilation at normal renal perfusion pressure has almost the same magnitude as that at $75 \mathrm{mmHg}$. whereas the renal vasodilation induced by glucagon is almost abolished at $75 \mathrm{mmHg}$.

We have previously reported that autoregulation-induced resistance alteration occurs predominantly at the afferent arteriole (14). Therefore, the observation that glucagon interferes selectively with the autoregulatory component suggests that glucagon primarily dilates the afferent arteriole. In contrast to the action of glucagon, adenosine increased RBF even at an RPP of $75 \mathrm{mmHg}$, having a minimum value of afferent arteriolar resist- 
ance. This observation indicates that adenosine may preferentially dilate the postglomerular efferent arterioles. In contrast, Osswald et al. (5) reported that adenosine preferentially constricts the afferent arterioles in the superficial cortex of the canine kidney by a micropuncture method. However, it is not reasonable to simply compare our results with theirs, since we $(19,20)$ and Macias et al. (22) reported that adenosine caused a flow redistribution from the outer cortex to the inner cortex. We can not rule out an afferent arteriolar constrictive action of adenosine based on the present results, but it is likely that the effect of adenosine on the afferent arteriole is negligible on the basis of the whole kidney and that adenosine at the dose of 20 $\mu \mathrm{g} / \mathrm{kg} / \mathrm{min}$ preferentially dilates the efferent arteriole in the whole kidney.

In the presence of efferent arteriolar dilation, one would have expected an increase in both RBF and GFR by superimposition of glucagon which selectively dilates the afferent arteriole. A similar pressure-flow relation would have been expected as it would be induced by $\mathrm{ACh}$ that dilates both afferent and efferent arterioles $(14,16,17)$. Renal vasodilation induced by $\mathrm{ACh}$ might be a maximum, since other vasodilators such as bradykinin and prostaglandin $E_{2}$ could not dilate the rena! blood vessels to a greater extent than the dilation that would occur with ACh (23-25). As shown in Table 1, the superimposition of glucagon to adenosine can induce further vasodilation and restore the GFR to the control value at normal RPP. In addition, superimposed glucagon completely abolished the RBF and GFR autoregulatory capability that was maintaineo during adenosine infusion, and there was no significant difference between pressure-flow and -GFR relations obtained during the infusion of $\mathrm{ACh}$ and adenosine plus glucagon. These findings may indicate that glucagon and adenosine selectively dilate the afferent arteriole and the efferent arteriole to the maximum extent, respectively.

Selective vasodilation of the afferent arteriole or the efferent arteriole would be expected to increase or decrease GFR to a greater extent than would occur with vasodilators that dilate both afferent and efferent arterioles. In fact, glucagon increased GFR and adenosine decreased GFR significantly at normal pressure. Our results confirm the data obtained in previous in vivo studies $(4,5,8$, 9. 21, 26). These GFR responses might be induced by the change in the glomerular filtration coefficient. However, since previous reports showed no effect of either adenosine (5) or glucagon (9) on the permeability coefficient of glomerular capillary, GFR responses may depend predominantly on the hydraulic pressure difference across the glomerular capillary wall, which is determined by the relative tone of the two resistance vessels. Even if both adenosine and glucagon do change the permeability coefficient, the RPP$G F R$ relation obtained during infusion of both agents may give us a great deal of information. The RPP-GFR relationship during infusion of glucagon or adenosine was qualitatively similar to the respective RPP-RBF relationship. This RPP-GFR relationship leads us to the same conclusion obtained from the RPPRBF relationship.

The present study clearly demonstrated that glucagon and adenosine preferentially dilate the afferent arteriole and the efferent arteriole, respectively. This action of adenosine may indicate its importance as a physiological controller of renal hemodynamics. An increase in adenosine concentration in renal tissue indicates a negative balance between oxygen consumption and oxygen supply. Adenosine should reduce the oxygen consumption in the kidney by reducing GFR and tubular reabsorption, and increase the oxygen supply by increasing RBF. Thus, we suggest that adenosine plays an importent role in linking the control of RBF and GFR to renal metabolism. In addition, both agents can be considered to be useful pharmacological tools for clarifying the regulatory mechanisms of renal hemodynamics.

Acknowledgments: This study was supported by Grant-in-Aid for Scientific Research 62570089 from the Ministry of Education. Science and Culture of Japan. Glucagon was the kind gift of Kodama Pharmaceutical Co. (Japan). We are grateful to Prof. Ian A. Reid (Univ. of California, San Francisco) for reading the manuscript, Chikage Kimura and Hiroko Irie for secretarial assistance, and Yuka Tsuruno for technical assistance. 


\section{References}

1 Osswald, H., Nabakowski, G. and Hermes, $H$.: Adenosine as a possible mediator of metabolic control of glomerular filtration rate. Int. J. Biochem. 12, 263-267 (1980)

2 Osswald, H., Hermes, H.H. and Nabakowski, G.: Role of adenosine in signal transmission of tubuloglomerular feedback. Kidney Int. 22, \$136 S142 (1982)

3 Spielman, W.S. and Thompson, C.I.: A proposed role for adenosine in the regulation of renal hemodynamics and renin release. Am. J. Physiol. 242 (Renal Fluid Electrolyte Physiol. 11), F423F435 (1982)

4 Tagawa, H. and Vander, A.J.: Effects of adenosine compounds on renal function and renin secretion in dogs. Circ. Res. 26, 327-338 (1970)

5 Osswald, H., Spielman, W.S. and Knox, F.G.: Mechanism of adenosine mediated decreases in glomerular filtration rate in dogs. Circ. Res. 43, 465-469 (1978)

6 Murray, R.D. and Churchill, P.C.: Concentration dependency of the renal vascular and renin secretory responses to adenosine receptor agonists. J. Pharmacol. Exp. Ther. 232, 189-193 (1985)

7 Premen, A.J., Hall, J.E. and Smith, M.J., Jr.: Postprandial regulation of renal hemodynamics: role of pancreatic glucagon. Am. J. Physiol. 248 (Renal Fluid Electrolyte Physiol. 17). F656-F662 (1985)

8 Ueda, J., Nakanishi, H., Miyazaki, M. and Abe, Y.: Effects of glucagon on the renal hemodynamics of dogs. Eur. J. Pharmacol. 41, 209-212 (1977)

9 Ueda, J., Nakanishi, H., Miyazaki, M. and Abe, Y.: Effects of glucagon on the renal hemodynamics in the dog. Japan. Circ. J. 41, 991-998 (1977)

10 Selkurt, E.E., Hall, P.W. and Spencer, M.P.: Influence of graded arterial pressure decrement of renal clearance of creatinine, $p$-aminohippurate and sodium. Am. J. Physiol. 159, 359-378 (1949)

11 Thurau, K. and Wober, E.: Zur Localisation der autoregulativen Widerstandsanderungen der Niere. Pflugers Arch. Ges. Physiol. 274, 553-566 (1962)

12 Schmid, H.E., Garrett, R.C. and Spencer, M.P.: Intrinsic hemodynamic adjustments to reduced renal pressure gradients. Circ. Res, 15, Supp. 1, 170-177 (1964)

13 Robertson, C.R., Deen, W.M., Troy, J.L. and Brenner, B.M.: Dynamics of glomerular ultrafiltration in the rat. III. Hemodynamics and autoregulation. Am. J. Physiol. 223, 1191-1200
(1972)

14 Abe, Y., Dixon, F. and McNay, J.L.: Dissociation between autoregulation of renal blood flow and glomerular filtration rate. Am. J. Physiol. 219, 986-993 (1970)

15 Navar, L.G.: Minimal preglomerular resistance and calculation of normal glomerular pressure. Am. J. Physiol. 219, 1658-1664 (1970)

16 Thomas, C.E., Ott, C.E., Bell, P.D., Knox, F.G. and Navar, L.G.: Glomerular filtration dynamics during renal vasodilation with acetylcholine in the dog. Am. J. Physiol. 244 (Renal Fluid Electralyte Physiol. 13), F606-F611 (1983)

17 Edwards, R.M.: Response of isolated renal arterioles to acetylcholine, dopamine and bradykinin. Am. J. Physiol. 248 (Renal Fluid ElectroIyte Physiol. 17). F183-F189 (1985)

18 Walser, M., Davidson, D.G. and Orloff, J.: The renal clearance of alkali-stable inulin. J. Clin. Invest. 34, 1520-1523 (1955)

19 Ueda, J.: Adenine nucleotides and renal function: Special reference with intrarenal distribution of blood flow. Japan. J. Pharmacol. 22, Supp. 5 (1972)

20 Ueda, J., Abe, Y., Okahara, T, and Yamamoto, K.: Adenine nucleotides and renal function: Special reference with intrarenal distribution of blood flow. Osaka City Med. J. 20, 33-50 (1974)

21 Premen, A.J., Hall, J.E., Mizelle, H.L. and Cornell, J.E.: Maintenance of renal autoregulation during infusion of aminophylline or adenosine. Am. J. Physiol. 248 (Renal Fluid Electrolyte Physiol. 17), F366-F373 (1985)

22 Macias, J.F., Fiksen-Olsen, M., Romero, J.C. and Knox, F.G.: Intrarenal blood flow distribution during adenosine-mediated vasoconstriction. Am. J. Physiol. 244 (Heart Circ. Physiol. 13). H138-H141 (1983)

23 McNay, J.L. and Abe, Y.: Redistribution of cortical blood flow during renal vasodilation in dogs. Circ. Res. 27, 1023-1032 (1970)

24 Stein, J.H., Ferris, T.F., Huprich, J.E., Smith, T.C. and Osgood, R.W.: Effect of renal vasodilation on the distribution of cortical blood flow in the kidney of the dog. J. Clin. Invest. 50 , 1429-1438 (1971)

25 Abe, $Y$., Kishimoto, $T$. and Yamamoto, $K$.: Relationship between renin secretion and renal autoregulation in the dogs. Japan. Circ. J. 40, 935-943 (1976)

26 Hall, J.E., Granger, J.P. and Hester, R.L.: Interactions between adenosine and angiotensin II in controlling glomerular filtration. Am. J. Physiol. 248 (Renal Fluid Electrolyte Physiol. 17). F340-F346 (1985) 